\title{
Blockchain-Based Equity and STOs: Towards a Liquid Market for SME Financing?
}

\author{
Florie Mazzorana-Kremer \\ INSEEC Research Center, International University of Monaco, Monte-Carlo, Monaco \\ Email: fmazzorana@inseec.com
}

How to cite this paper: Mazzorana-Kremer, F. (2019) Blockchain-Based Equity and STOs: Towards a Liquid Market for SME Financing? Theoretical Economics Letters, 9, 1534-1552.

https://doi.org/10.4236/tel.2019.95099

Received: April 12, 2019

Accepted: June 18, 2019

Published: June 21, 2019

Copyright (c) 2019 by author(s) and Scientific Research Publishing Inc. This work is licensed under the Creative Commons Attribution International License (CC BY 4.0).

http://creativecommons.org/licenses/by/4.0/

(c) (i) Open Access

\begin{abstract}
Security Token Offerings (STOs) are a very recent phenomenon that has started to replace the Initial Coin Offering (ICO) one for financing companies through blockchain networks. Contrary to ICOs, which are based on "utility tokens", STOs issue "security tokens" that are likely to achieve revenues in the same way that bonds or shares do. However, because they utilize the blockchain network, they are expected to benefit from lower intermediary and transaction costs. The objective of this paper is to examine, for the first time in financial research, to what extent this nascent market can become a liquid one, adapted for small and medium-sized enterprises (SMEs). To address this still unexplored issue, we proceed in two stages. First, we develop the technical characteristics of security tokens. Then, we analyze the trading volumes of a very few ones, although it has proved difficult to conduct a relevant empirical analysis. Our results are that, as for ICOs, the technical nature of security tokens can greatly facilitate their listing and exchange. However, there are significant disparities in their use and, for the moment, most of them remain locked in the wallet of so-called accredited investors. As a result, the potential of the blockchain-based equity market is still uncertain: STOs are likely to represent a growing and liquid alternative to IPOs, private equity and crowd funding to finance SMEs. Nevertheless, the liquidity of their digital assets strongly depends on the quality of their issuers and on the existence of specialized trading platforms.
\end{abstract}

\section{Keywords}

Blockchain, Equity, Financing, SMEs, Security Tokens, STO, ICO, Liquidity, Funding

\section{Introduction}

In our present economy, the choice of an equity funding solution plays a deter- 
minant role for companies, especially in the small and middle cap sector, in which fundraising costs can quickly become prohibitive.

Indeed, small- and medium-sized companies often have a limited access to equity and bank lending remains their most common source of financing. Most of the time, equity capital markets are fragmented and not highly attractive to small and medium-sized enterprises (SMEs), with low levels of cross-border investment [1] and low liquidity.

In 2008, the launch of the bit coin digital money by Satoshi Nakamoto [2] made public a new and tremendous underlying technology: the blockchain: it enables the exchange of value on the internet in a trusted and secure manner. The world had known the Internet of information. It was about to discover the Internet of value [3].

Taking advantage of this innovation, several years after, a few companies or project teams started to raise money with an incredible ease and speed, pacing the way to a new kind of fundraising practice. They were doing what we call an "Initial Coin Offering" (ICO), collecting crypto currencies in exchange for "utility tokens" that were giving rights to their future services of products. After a tremendous success, ICOs have started to slow down in number and volume because of the lack of regulation and protection they offered to investors. As an answer to these issues, "security tokens" have started to emerge, i.e. digital units that are deemed real rights on companies' ownership or revenues, and thus have to comply with the appropriate financial regulations. ICOs have been progressively replaced by Security Token Offerings (STOs), but the phenomenon is still young and the implementation of the latter sounds a bit more complex.

Thanks to this new type of fundraising process, blockchain offers SMEs a unique opportunity to get funded in a very competitive way. Constituting a first attempt to fill the literature gap on this hot topic, this study tries to answer the following question: to what extent can STOs take the lead over current equity financing solutions and offer a more liquid market for investments in SMEs?

More precisely, this paper is structured in three main parts. In the first part, it develops the different sources of equity financing for small- and medium-sized companies and analyzes their main advantages and disadvantages. It also describes and compares these traditional sources with their blockchain-based alternative.

Secondly, the paper makes a brief literature review on this issue although, this one remains scarce.

Then, in order to answer the research question, it analyzes the structure of current security token sand tries to determine if they are made to become liquid instruments. It also analyzes the very few STOs that already have historical records of trading and reviews their traded volumes. Indeed, the STO market is still nascent and as for now there is almost no historical data of volume and price available. Most STOs are under a year old and their security tokens still have to be kept by their primary investors for regulatory purpose. 
The main outcomes of this study are that security tokens are conceived in a way that should make them easy to list on exchange places. However, most of these exchange platforms are still under conception and the lock-up period imposed my most financial regulators makes it is impossible, at this stage, to conduct a detailed empirical study. It nevertheless offers interesting opportunities for future research on this field. In addition, the preliminary data available for analysis already show a great disparity between volumes exchanged from one security token to another. This shows that the liquidity of a security token also depends on the quality and the communication of its issuer.

\section{Blockchain-Based Funding versus Traditional Sources of Equity}

This section first develops the existing sources of equity financing and presents their advantages and disadvantages. Then, it compares these traditional sources of financing with blockchain-based solutions such as Security Token Offerings.

\subsection{Review and Limits of Current Sources of Equity Financing}

One traditional source of equity for SMEs and even large cap companies is private equity. It offers a well-known opportunity for unlisted companies to get funded. However, it often compels their shareholders to accept an important ownership dilution and a strong external control on their management. Moreover, unlike the stock market, private equity expects compensation for the significant asymmetry of information that exists on the unlisted market; the category of investors it attracts generally expects a higher cost of capital and requires access to a substantial amount of data on the potential investee companies to conduct detailed due diligence. In addition, the costs incurred by these investigations are "rein voiced" to the company after completion of the investment. It is, thus, a long and expensive procedure for SMEs, with no guarantee of success.

Faced with the difficulties and disadvantages of this option, crowd funding has grown at a fast rate in the past few years. The concept of "crowd funding" refers to the process of raising money from many individual donors-the "crowd"-essentially through the internet, in exchange for some form of reward or voting rights [4].

The first crowd funding platform, Artistshare.com, was created in 2003. S and since then, the number of similar platforms and and the equity crowd funding transactions have has kept rising (See Figure 1).

Over time, crowd funding has become an alternative source of funding across many other sectors and has a potential to change the way SMEs are financed. The increasing number of platforms that have emerged since 2010 offers a different audience of investors, creating a possible "Big Bang Disruption" of the equity financing market [6]. Crowd funding generally represents a smaller risk of ownership dilution, as well as an often quicker and cheaper way to achieve funding objectives. 


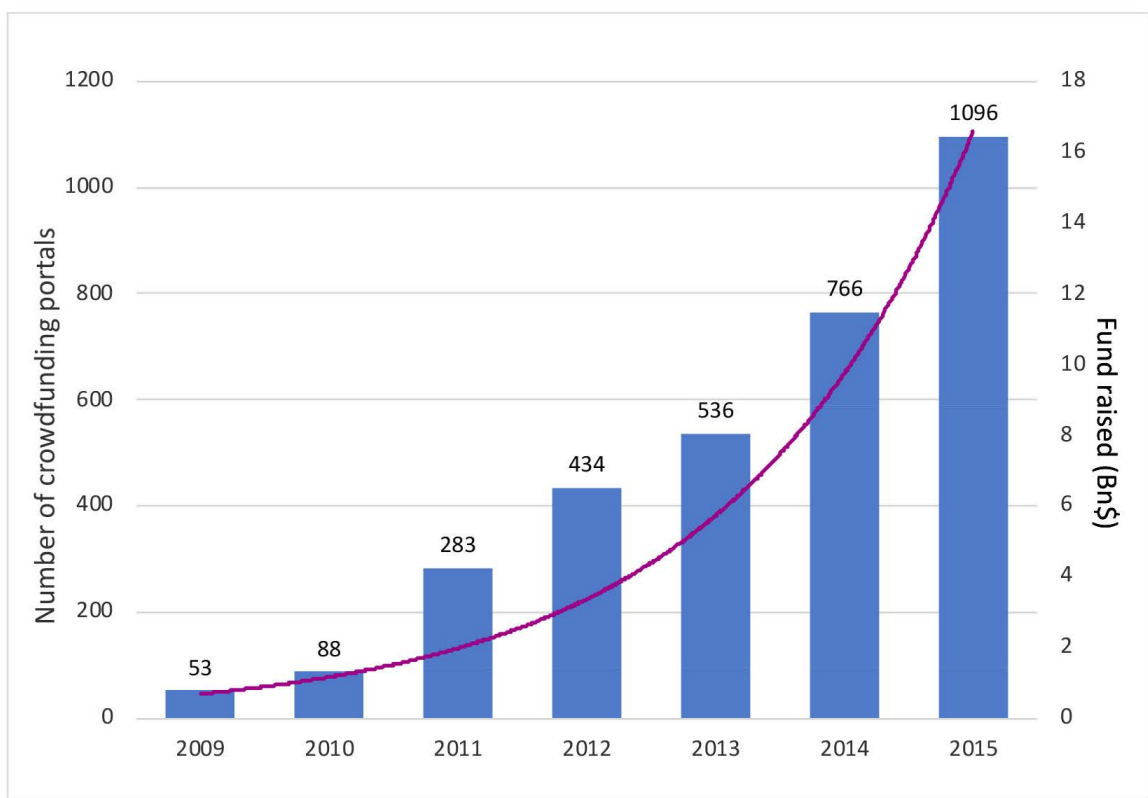

Figure 1. The global crowd funding market: raised funds and number of portals. Source: adapted from forbes \& schaefer, 2017 [5].

Nevertheless, such alternative has shown important limits and disadvantages during the recent years. First of all, capital raising on specialized platforms must becom pliant with national regulations. Thus, platforms have to perform tailored and tiresome compliance work, and its related implementation cost is indirectly charged to companies through high intermediary fees. Another major issue of this funding process-maybe the most important one-is the absence of secondary liquid markets [7].

According to Keynes [8], an asset can be considered as more liquid if it is more likely to be resold at a short notice without loss. In crowd funding, the liquidity risk that investors face relates to their difficulty to exit an investment prior to its maturity. A note from the Financial Conduct Authority in 2013 highlights this fact: "Consumers investing in such equity need to understand that they will probably have to wait until an event occurs, such as the sale of the company, a management buy-out or a flotation, before getting a return" [9]. One technological explanation that can be given for this phenomenon is that there are over 450 crowd funding platforms worldwide, and each one has its unique, often arbitrary rules, regulations, limitations and characteristics [10]. Thus, investments made in companies, on a specific portal, are unlikely to be resold to other investors on other portals.

The size of equity crowd funding in Europe was estimated to be in the range of EUR 50 - $100 \mathrm{M}$ in 2013, a rather small amount compared with the EUR 26B value of the IPO market [11]. This is not surprising considering that $50 \%$ of executives believe that the most important factor that must be retained when choosing a stock exchange is liquidity (See Figure 2). Compared to private equity and crowd funding, traditional stock markets still present serious advantages. 


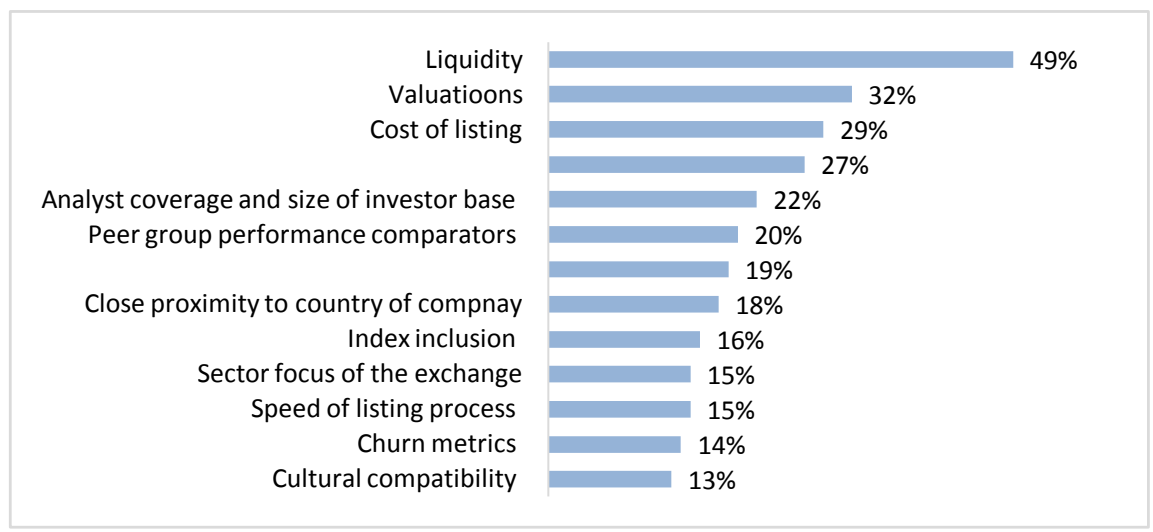

Figure 2. Most important factors when choosing a stock exchange/market for an IPO. Source: Extract from the Economist Intelligence Unit 2018 Survey, ordered by PwC [12].

On organized stock markets, private companies can decide to go public and make what we call an "Initial Public Offering" (IPO), i.e. a first sale of shares to the public. Preliminary conditions they must fulfill before going public are to prove their financial strength, market potential growth and good record history. They also have to perform regular and transparent financial records.

Compared to other sources of equity, stock markets present numerous advantages. The largest ones, such as Nasdaq, Euronext, the New York Stock Exchange and the Frankfurt Stock Exchange are very liquid. Such liquidity is important because it increases attractiveness to issuers and investors, as this translates into greater use of the market, greater confidence, and greater ability to attract new stakeholders that see opportunities to exit at any time, under transparent conditions. On stock markets, liquidity can be broadly understood as "the ability to facilitate large volumes of trade without causing excessive price movements, while still reflecting a steady and fair market price" [13].

However, not all companies are going public on liquid stock markets. Some simply cannot while others weigh expected benefits against the costs. Therefore, it is crucial to look closely at the costs of listing.

In most cases, going public is an expensive process under which a company often hires attorneys, auditors and underwriters to prepare financial statements and regulatory filings and to interact with regulators and investors during the IPO.

The cost of an IPO, including listing fees and advisor fees, typically runs in the hundreds of thousands of dollars and can exceed US\$1M [14].

To offer an alternative to the largest organized stock exchanges, a large number of SME public equity markets have been created since 1970. However, most of these exchanges failed to attract sufficient companies for listing or to achieve sufficient trading to maintain active markets. Some of the difficulties encountered by the companies that have opted for these new kinds of market places are 1) high listing and maintenance costs, 2) an important administrative and regulatory burden, and 3) an insufficient preparation to adapt their management 
practices in an appropriate way for a listed company. A good example of thatis the European market of SME Exchanges which only represented 30\% the liquidity of main markets in 2014 [15].

In addition, regarding the specific cost of issuing for SMEs, a study performed by Oliver Wyman assumes a range of $\$ 80-100 \mathrm{~K}$ for smaller companies plus $\$ 100$ - $120 \mathrm{~K}$ per year to remain listed. Therefore, altogether, stock exchanges for small and medium-sized companies still represent important listing costs and a small liquidity for their investors.

\subsection{Security Tokens, STOS and Blockchain-Based Equity Funding}

In 2008, the first cryptocurrency, bitcoin, was created by a so-called Satoshi Nakamoto [2]. Such currency relied on a revolutionary technology—blockchain—which made the exchange of value with no intermediary in a trusted and secure way possible for the first time in the history of the Internet. A few years later, other public blockchains were launched, such as Ethereum, in 2013, which had the ambition to propose more than just a cryptocurrency. Indeed, Ethereum is based on a protocol that uses a Turing-complete programming language, which enables the exchange of a wider range of valuable instruments [16]. Since then, the financing industry has started to envisage additional applications of this new technology.

As a result, approximately ten years after the Bitcoin invention, several platforms have started proposing the trading of digital assets, such as the Ethereum-based Dx. Exchange since March 14th, 2019. Things are moving fast in the blockchain-based financial world: Dx. Exchange already offers a few tokenized stocks and ETFs, and other similar portals such as Polymath, tZERO, OpenFinance Network, Ledgity, etc. have just been or are about to be launched.

Let's now go over the details of blockchain and some of its key concepts:

Blockchain, also called "Distributed Ledger Technology", is the underlying technology of Bitcoin [2]. It can be described as an ever-changing database which stores the history of the exchange made between its users and which is shared on a peer-to-peer network. Changes on the database are made by active network participants through a consensus algorithm and are incorporated in new blocks. These blocks are usually issued at a steady pace and made immutable through cryptography.

Originally, this technology was only used to record digital money transactions such as Bitcoin [17], but it quickly became clear that its capabilities were broader. Indeed, examples of its other applications are: currency, smart contracts representing financial assets, and social functions such as notary, voting and healthcare applications [18].

It is relevant to mention that there are several types of blockchains. Some are public-like Bitcoin, Ethereum, Mastercoin, Litecoin-and generally rely on a proof of work consensus. In those types of blockchains, anyone can join the network, use its protocol and become one of the "nodes" that write the new 
blocks and validate the transactions. There are also private or consortium blockchains, like Ripple, EOS, Stellar, etc. On these blockchains, only authorized nodes can read or write new blocks. Suchalternative systems generally have lower costs and faster speeds than public blockchains, while providing a lower level of security and decentralization.

Most blockchains have a native token that incentivizes nodes to validate transactions [19] or that represent the unit of value of their transactions. Two famous examples of tokens are bitcoin and ether, the native tokens of the blockchains that have the same name. Other tokens are digital units issued by smart contracts on an existing blockchain.

According to the Swiss Financial Market Supervisory Authority (FINMA), three kinds of tokens can be distinguished: payment tokens, utility tokens, and security tokens [20]. Payment tokens are described as cryptocurrencies that "are intended to be used, now or in the future, as a means of payment for acquiring goods or services or as a means of money or value transfer"; utility tokens are said to "provide access digitally to an application or service by means of a blockchain-based infrastructure"; and finally, asset tokens, also called security tokens, "represent assets such as a debt or equity claim on the issuer".

Over the very last years, many startups have offered utility tokens in exchange for money or cryptocurrencies to finance their projects. They have done what we call an "Initial Coin Offering" (ICO), i.e. a swap of newly created tokens with cryptocurrencies or fiat money [19]. In an ICO, investors transfer funds to the start-up and receive, in return, tokens of a blockchain specifically created for the ICO or through a smart contract on an existing blockchain.

The ICO phenomenon has known an impressive success during the years 2017-2018, partly thanks to the emergence of a simple and reproducible token model: the ERC20. Such standard was proposed at the end of 2015 on GitHub by a participant of the Ethereum community in order to facilitate the creation and exchange of tokens on Ethereum [21]. Its simple code was "copied/pasted" by many developers who were able to easily and quickly issue the tokens of their own ICOs. The fact that most companies were using the same standard also greatly facilitated the listing of their tokens on trading platforms such as Kraken or Coinbase.

However, most of these ICOs were only offering utility tokens. As nothing tied down their value, they rapidly became very volatile and unsecure for investors, unlike conventional stocks and securities [22]. As a result, after 2018, this type of fundraising significantly declined (Figure 3).

Despite this, for most start-ups, the ICO has clearly demonstrated that blockchain technology can be a very competitive alternative for funding, presenting several advantages compared to traditional financing.

First, unlike crowdfunding, ICOs offer potentially liquid instruments that could be resold on many portals. Secondly, the issuing of tokens for fiat money or cryptocurrencies is a cheap and simple process. Compared to IPOs or 


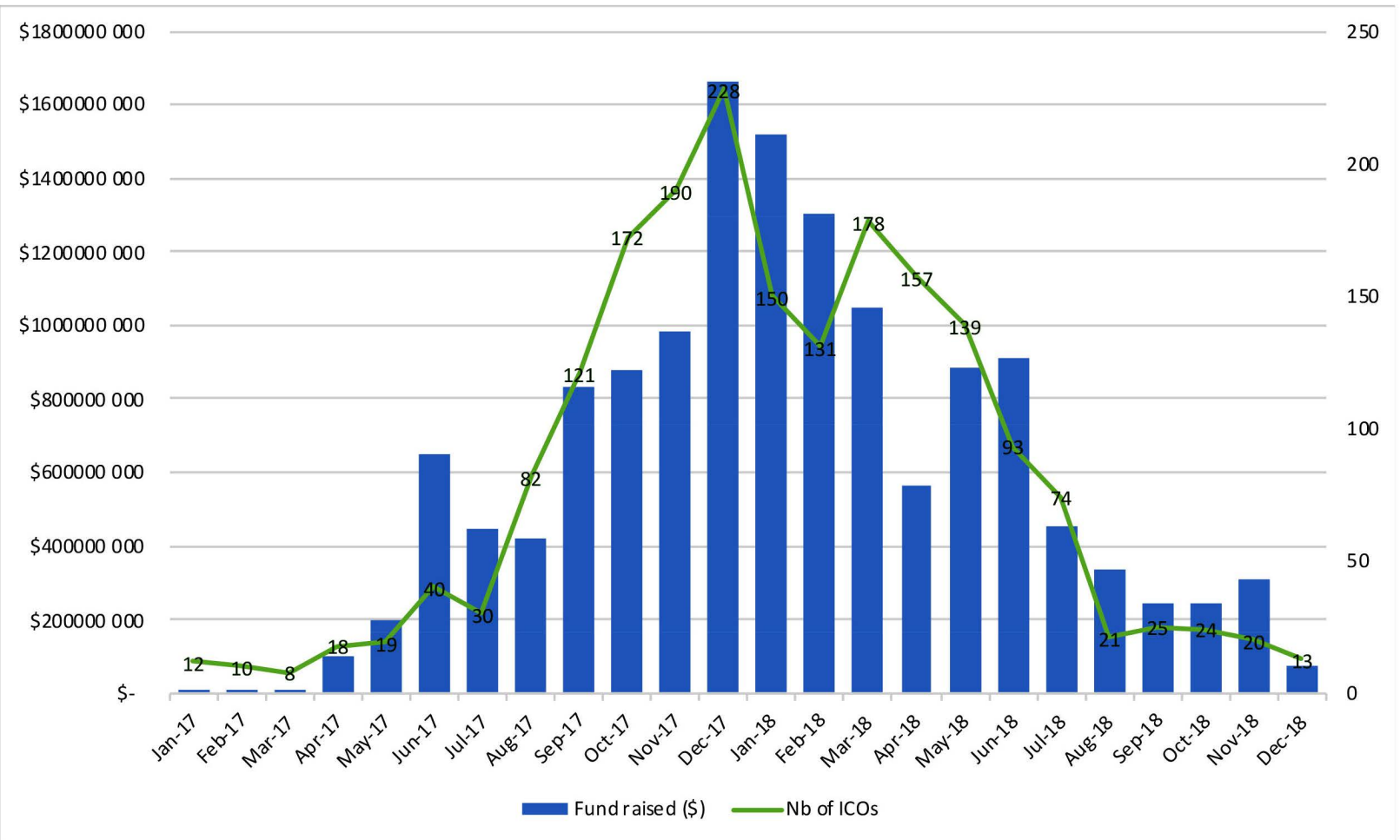

Figure 3. Fund raised and number of ICOs over the 2017-2018 period. Source: adapted by author from icodata.io.

fund-raising with private equity firms, these operations are virtually cost-neutral, and no intermediary is required. Nevertheless, with no ownership or right on the profit to their holders, utility tokens are not subject to regulation. This greatly limits the administrative burden and costs incurred by companies for their ICO campaigns. On the other hand, with very little guarantee of profit sharing, this also explains why ICOs have drastically reduced in number and volume.

Today, to address these issues and the lack of investor protection, some "crypto" startups choose to offer security tokens. Unlike utility tokens, they consist of real rights to corporate ownership or income. They still represent only $\$ 400 \mathrm{M}$ of funds raised [23], far behind the $\$ 20 \mathrm{~B}$ of the ICOs, for a few reasons. The phenomenon is very new; security tokens must comply with financial regulations; there is still little legal history on the subject; and thus, their implementation sounds a little more complex than utility tokens.

From now on, the potential of blockchain technology for issuing and trading digital assets is no longer an issue. Key institutional players such as the stock exchanges of London, Boston, New York, Gibraltar, Malta, Singapore and Switzerland recently confirmed their interest in the technology and reiterated their efforts to enable the enlisting of tokenized securities on their platforms. In addition, market participants tend to be more and more convinced by the funding potential of blockchain. To illustrate this phenomenon, a survey conducted by the Economist Intelligence Unit in 2018 [12] among 370 equity funding participants showed that for $24 \%$ of them, the crypto market was one of the most at- 
tractive options for private financing (Figure 4). This reflects a burgeoning but growing interest in blockchain-based financing solutions.

In this context, the issue of blockchain-based equity financing for startups and SMEs seems particularly interesting. The next part of the article develops what research literature, still rare, says on the subject.

\section{Literature Review}

The literature paying attention to the use of blockchain for equity funding is still scarce. One of the first papers on the question is the one by Zhu and Zhou [17], which focuses on China. According to its authors, blockchain technology has good chances of resolving some of the current problems of crowdfunding: traditional registration processes of investors are long and expensive, shareholders are scattered over wide regions and ownership transfers are complex at the operational level. They believe blockchain-based equity funding will facilitate crowdfunding equity circulation and help address their financial security and regulatory compliance. Indeed, blockchain-based shareholder lists can be used as a better alternative to paper documentation or centralized storage media. It could enable "crowdfunding shareholders located in different regions to securely register their rights at low cost", and a smart contract on a public blockchain could make their rights on a company automatically recognized. Authors thus expect blockchain to reduce administrative costs (low-cost equity registration, transaction and transfer).

In a more recent paper (2019), Ante \& Fielder [24] compare STOs with ICOs and share the opinion that "offerings of security tokens promise to better fit firms' and investors' needs and fill the role that ICOs failed to meet". They mention several expected advantages of security tokens over traditional stocks: they are immediately transferable, they can be traded $24 / 7$ on secondary markets, they no longer require brokers and custody accounts, and their underlying blockchain ensures the transparency and security of transactions.

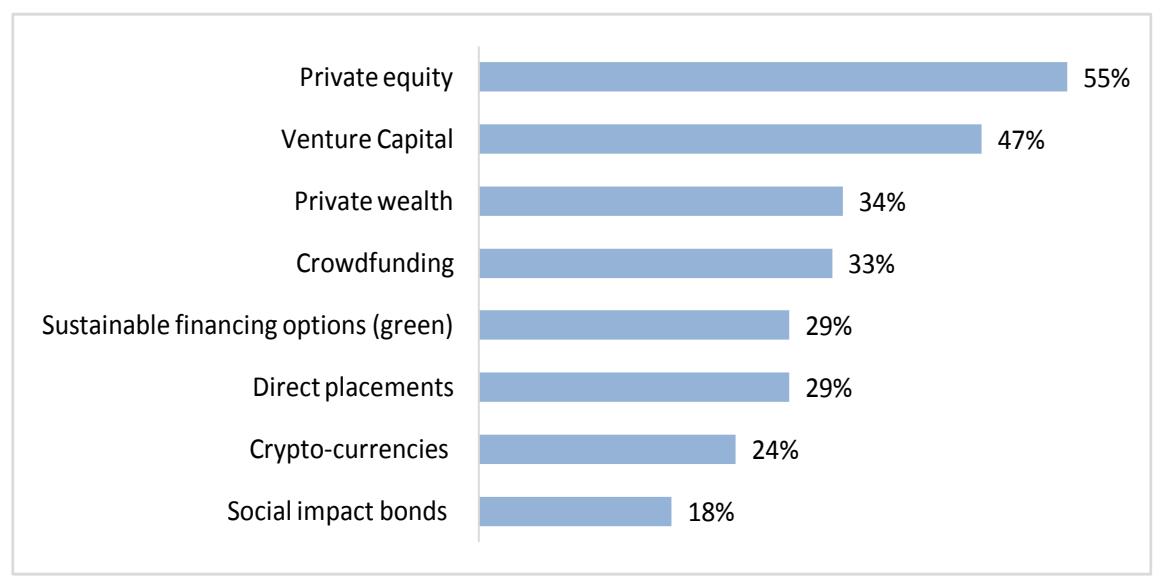

Figure 4. Which private financing options do you find most attractive? Source: Extract from the Economist Intelligence Unit 2018 Survey, ordered by PwC [12]. 
Another advantage seen in blockchain and quoted in a report from the Division of Research \& Statistics and Monetary Affairs of the Federal Reserve Board [25], is its capacity to greatly shorten the current 3-day settlement time required for equity transactions on exchanges. However, some technical and administrative constraints should also ideally be included in future settlement solutions to solve current loss issues of cryptocurrencies. Indeed, many users of crypto-tokens suffer immediate and irrevocable losses without recourse when their keys or access credentials are lost or compromised.

On the other hand, existing research also highlights some problems related to security tokens [24]:

First, the regulatory framework for security tokens (that aim to be "global", on a distributed ledger) is still unclear: the SEC uses the Howey test to determine whether a token is a security or not while the EU regulations on the topic are still pending, although some countries like Germany have already ruled;

Secondly, even if the biggest promise of security tokens is that the liquidity observed for ICOs may be carried over to the market for STOs, a liquid market still requires existing infrastructures and firm quality [26].

The only empirical study we found on STOs is the one of Ante \& Fielder who claim to have analyzed a dataset of 151 security tokens. We think that our subject is slightly different from theirs given that-in our opinion-most of the offers they have been able to review do not represent "intentional" STOs. Indeed, many of the companies that launched their ICO before 2018 did not even realized that the tokens issued could be seen as securities by financial regulatory authorities. For instance, the tokens issued by the DAO organization in April 2016 on Ethereum have been considered as so by the Securities and Exchange Commission (SEC) in 2017 [27]. Many other US companies were concerned by the SEC rules without knowing and, in 2017, none had registered as a security offering. Some, like the startup Munchee, were even fined and forced to refund buyers because they should have limited their sales to accredited investors. Therefore, including such ICOs in an empirical analysis of STOs seems inappropriate to us since neither their issuers nor their investors knew they had traded securities.

Unfortunately, most STOs that can be characterized as intentional, and that clearly implement profit sharing solutions, are less than a year old. This greatly limits the possibility of empirical analysis.

On the ICO side, most research papers focus on what makes ICOs successful [24] [28] [29] and are interested in their communication in social networks, the nature of their services, patents and transparency, etc. It is relevant to mention that some of these papers only focus on utility tokens while others partly include security tokens in their analysis of ICOs.

A study by Fisch [29] relying on a sample of 238 ICO campaigns finds that white papers, source code quality and the use of the Ethereum-standard are determinants of the amount raised, while venture characteristics are less relevant. Another one from Conley [22] provides "good practices" for issuers to support 
and boost the value of their tokens. This last research also advises issuers to make their value proposition clear to increase investors' willingness to pay and thus improve the liquidity of their digital assets.

Some papers also compare ICOs to IPOs [30] [31], evidencing a high information asymmetry on the ICO side, partly compensated by white papers and active social media communication for the most successful ones. As a result, the current stagnation of the ICO market is often explained by a lack of regulation to protect investors and guarantee the disclosure of some valuable information regarding the companies. In the literature reviewed, it also appears that ICOs differ from IPOs in terms of target proceeds, fraction of total token supply sold, distribution method, lock-ups and token rights. However, like IPOs, some ICOs offer or require lock-up periods, during which ICO participants may not sell their tokens, but they do not have their underwriting and disclosure costs that represent a significant fraction of the funds raised. Existing ICOs with the highest level of liquidity also consistently tried to reduce information asymmetry [31].

In short, since firm size, trading costs, information supply, stock returns and risks influence liquidity [32], STOs are likely to be a very promising market: they are bound to provide lower transaction costs than IPOs; a more secured regulatory environment and more rights on profit than ICOs; they should comply with information disclosure obligations... and they are likely to solve the liquidity issue of equity crowdfunding on current platforms. Therefore, blockchain appears to be an interesting alternative way for startups and SME to get financed.

However, existing literature does not specifically study the recent and intentional offers of security tokens. Nor does it pay attention to the underlying blockchain used by issuers and platforms. Therefore, this might condition the future level of circulation of security tokens between investors.

In the next parts of this article, we analyze current STOs, the nature of their tokens, and underlying blockchain-networks they use. We try to figure out if they can benefit from the same favorable technical environment than ICOs. In addition, we analyze a dataset of ICOs-that were not full spams-and we use a measure called velocity to assess their liquidity.

\section{Methodology}

As previously explained, the 2017/2018 ICO success-in terms of funding and liquidity - was partly caused 1) by the existence of specialized platforms and information websites and 2) by the ERC20 standard, which limited implementation costs and efforts for token issuance and listing. Furthermore, almost all ICOs used the same communication channels such as Reddit, Bitcointalk and Crunchbase, making it easy for investors to identify token "primary issuances" without a financial intermediary being necessary. At that time, US regulations were not yet in place to limit token issuers' subscriptions to accredited investors and to force investors to keep their tokens for a minimum period of time. 
The success of STOs can be expected to depend on similar factors: standardized technical rules, easy listing on exchange places, existence of good communication channels, secondary markets and tokens' accessibility to a wide range of investors.

As a result, the first part of our research describes and develops on the technical nature and environment of security tokens: do they follow a same kind of standard? Are there platforms in place to trade them? Are they accessible to a large number of potential investors? How should this recent market evolve?

To address this issue, we exploited publicly available information: STOs were identified using listing sites like CoinList.co, Token. Security, Tokenmarket.net, STOscope.com, and ICObench.com.

In a second research phase, we focused our analysis on a few STOs, for which token trades were not, or no longer, limited to accredited investors. During our investigation, it appeared that a very small number of successful STOs matched this criterion. We collected data on these scarce companies and their tokens from the website CoinMarketCap.com, although there have been several recent criticisms made about this data provider, which we will address further down. This work was limited by the fact that only a few coins had a trading history.

For these few tokens, we assessed the annual "token turnover" (TT). This is calculated by dividing the number of tokens traded over a year by the total supply of tokens during the same period.

$$
T T=\frac{\text { Total number of tokens traded }}{\text { Number of issued tokens }}
$$

Such proposed methodology and formula is close to the share turnover one, used for assessing stock liquidity:

Share turnover $=\frac{\text { Total number of shares traded }}{\text { Outstanding shares }}$. However, we do not apply it to

a specific stock exchange but to a specific token. Therefore, the information it provides is different, although giving us some indication of the token liquidity across available trading portals.

To illustrate the application of this methodology, let's take the NEXO security token, for which we have 11 months of historical trading data. There are 1,000,000,000 outstanding tokens and 560,000,011 tokens circulating in the public market; there has also been a total of $1,078,360,120$ trades in USD, which corresponds to $11,612,296,881$ tokens resold since the 1st of May 2018. Therefore, the token turnover ratio of NEXO has been: 11,612,296,881/1,000,000 $=11.61$ over 11 months, which leads to 12.7 over one year. In sum, the NEXO turnover for the year equals 13 times, which is quite high for a new market and nascent business.

Another equivalent measure of market liquidity is the token turnover velocity. On traditional stock markets, the turnover velocity is the ratio between the Electronic Order Book (EOB) turnover of domestic shares and their market capitalization. The value is annualized by multiplying the monthly average by 12 . 
In this research, we opted for calculating the annual token turnover, but we had little historical data and irregular historical periods to refer to. We did not calculate the turnover velocity (that is equivalent) because we believe that it would be more appropriate to use this ratio for a single market (for example, to assess the liquidity of the Binance platform).

For our calculations, we used the data from the website CoinMarketCap.com. We sometimes had to adapt it to our research needs; for example, the calculation of the market capitalization on the website only takes into account circulating tokens. Instead, we used the total number of tokens issued to calculate it. In the next section, we present the results of our research.

\section{Data Analysis and Results}

\subsection{Technical Analysis}

Between 2016 and 2018, most ICOs were launched on the Ethereum platform (Figure 5). Those who used the ERC20 standard were thus quickly listed on marketplaces. As they issued utility tokens, they were not subject to any regulation and had no restrictions on when and how these tokens could be resold, making them very liquid instruments.

Up to now, STOs have raised approximatively $\$ 400 \mathrm{M}$ [23]. The most renowned STOs are: tZERO ( $\$ 134 \mathrm{M}$ raised), Nexo, a crypto-based loans platform (\$52.4 $\mathrm{M}$ raised), and LDC by Lottery.com (\$65 M raised). They propose concrete dividends or similar profit-sharing mechanisms (tZERO will pay $10 \%$ of adjusted gross revenue to token holders on a quarterly basis; holders of NEXO tokens share $30 \%$ of the company's profits, paid monthly).

Our review of specialized websites ${ }^{1}$ helped us identify approximatively 40 "intentional" STOs that had successfully completed their fundraising, of which very few were "digital actions" like tZERO or Mt Pelerin. At the time of this research, 17 additional offerings of tokens representing equity were also being processed.

We focused our preliminary analysis on the following companies and STOs (Figure 6), the only ones for which we managed to collect a reasonable amount of information.

The outcome of this preliminary research shows that, as with ICOs, the majority of STOS use the Ethereum blockchain. Indeed, in our dataset, only three of them use another blockchain (the Swarm platform for the Robinhood Equity Token, and the Bizshake platform for the ART token and the NEO token. It is interesting to mention that Bitbond-which is not in our table, but is currently engaged in a STO-has chosen the Stellar blockchain. Bitbond sells digital bonds, not shares, but this example gives us a good idea of the options available for companies to issue their tokens.

\section{Blockchain technology and standards used for STOs}

Even if Ethereum remains the most popular blockchain in STOs, some companies are increasingly veering towards other solutions for scalability reasons. ${ }^{1}$ We mainly used the Internet for our research, through Google and websites like STOscope.com, ICObench.com, Coinlist.co and token.security. 


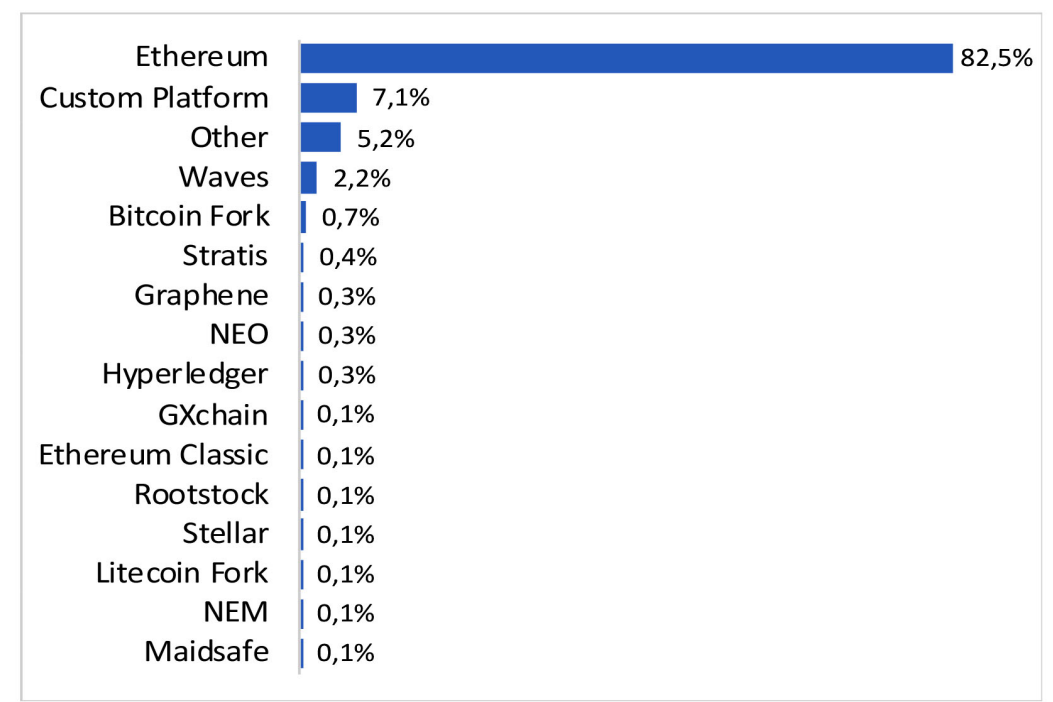

Figure 5. Blockchain platforms' market shares in ICOs. Source: icowatchlist.com, March 2019.

\begin{tabular}{|c|c|c|c|c|c|c|c|}
\hline $\begin{array}{l}\text { Project or } \\
\text { Company }\end{array}$ & $\begin{array}{l}\text { Fund raised } \\
\text { (in M\$s) }\end{array}$ & $\begin{array}{c}\text { Closing } \\
\text { date }\end{array}$ & Regulation & Country & $\begin{array}{c}\text { Security } \\
\text { Token type }\end{array}$ & Blockchain & $\begin{array}{l}\text { Market } \\
\text { activity }\end{array}$ \\
\hline tZero & 134,0 & Aug-18 & KYC, AML, Whitelist & US & ERC20 & Ethereum & Restric. Trade* \\
\hline Property coin & 50,4 & Jul-18 & KYC, AML, Whitelist & US & ERC20 & Ethereum & No trade \\
\hline Nexo & 52,5 & Apr-18 & KYC & SWITZ. & ERC20 & Ethereum & Trade \\
\hline Desico & 1,0 & Nov-18 & KYC, AML, Whitelist & LITH. & ERC20 & Ethereum & No trade \\
\hline 22X Fund & unknown & Mar-18 & KYC, AML, Whitelist & US & ERC20 & Ethereum & Restric. Trade* \\
\hline $\begin{array}{l}\text { Lottery.com (LDC } \\
\text { Crypto) }\end{array}$ & 65,0 & end-2018 & Unknown & US & DSO & Ethereum & Restric. Trade* \\
\hline Minthealth & 0,2 & Sep-18 & KYC, AML, Whitelist & US & ST20 & Ethereum & No trade \\
\hline BR11 & unknown & Dec-18 & None & CAYM. ISL & ERC223 & Ethereum & Not listed \\
\hline Mt Pelerin & 2,0 & Dec-18 & KYC, AML, Whitelist & SWITZ. & ERC20 & Ethereum & Not listed \\
\hline Gainfy & 1,0 & Dec-18 & KYC, AML, Whitelist & US & ERC223 & Ethereum & No trade \\
\hline Scientificcoin & & Feb-19 & KYC & US & ERC20 & Ethereum & No trade \\
\hline BizShake & 3,0 & Oct-18 & KYC, AML, Whitelist & US & NEP5 & $\mathrm{NeO}$ & No trade \\
\hline Corl & unknown & Oct-18 & KYC, AML, Whitelist & Canada & ST20 & Ethereum & No trade \\
\hline Sports Ledger & unknown & Feb-19 & KYC & UK & ERC20 & Ethereum & Not listed \\
\hline Bolton Coin & 67,0 & ongling & KYC, AML, Whitelist & CAYM. ISL & ERC20 & Ethereum & Not listed \\
\hline $\begin{array}{c}\text { Robinhood Equity } \\
\text { Token }\end{array}$ & unknown & Jan-19 & KYC, AML, Whitelist & US & SRC2O & $\begin{array}{l}\text { Swarm Fund } \\
\text { Private BC }\end{array}$ & No trade \\
\hline Jinbi & 47,0 & Dec-18 & KYC, AML, Whitelist & Belarus & ERC20 & Ethereum & Trade \\
\hline The Art Token & 11,0 & Dec-18 & KYC, AML, Whitelist & US & SRC20 & $\begin{array}{l}\text { Swarm Fund } \\
\text { Private BC }\end{array}$ & No trade \\
\hline
\end{tabular}

Figure 6. Regulation, token standard \&underlying blockchainsin 18closed STOs. Source: Author, April 2019.

Indeed, the Ethereum network can handle approximately 15 transactions per second; EOS, with its Delegated Proof of Stake consensus limited to 21 authorized nodes, carries out 4000 transactions per second [33], and the Stellar blockchain already handles 1000 transactions per second. As a result, it appears that a rising number of ICOs now choose EOS to issue their tokens, while a few STOs opt for Stellar.

For a liquid securities market, it is necessary to choose a scalable blockchain, capable of supporting a large number of transactions per second. However, because of its existing standards and compatibility, Ethereum still seems to be the 
first choice, even for security tokens.

However, the current ERC20 standard does not address many of the issues raised by security tokens. As a result, developers are working on new standards; a first one called ST20 has been proposed by Polymath. Based on the ERC20, it provides additional transfer restrictions. This standard has evolved and been replaced by a suite of programs called ERC-1400, which includes separate standardized modules on Ethereum [34]. This suite aims to address most of issues raised by security tokens. For instance, it includes the white listing of addresses for transfers (in the ERC20 model, transfers are blocked only if the account balance is insufficient) as well as:

- The ERC-1643 module for document management;

- The ERC-1644 for controller operations (for instance: executing court orders, solving problems related to the loss of private keys);

- The ERC-1594 for several core security issues, such as input of authorizations required from the real word and limitations to accredited investors;

- The ERC-1410 for incorporation of special features, such as time locks and voting privileges.

It is relevant to mention that other guidelines and models for security tokens have been developed. Securitize, a primary issuance platform, proposes the DS Protocol, built on Ethereum. It is designed to resolve liquidity and compliance issues. The security token platform OpenFinance also proposes its standard, the S3; Harbor follows in their footsteps with its R-Token protocol.

However, all of these protocols are built on Ethereum.

Outside Ethereum, a few STOs have used the Swarm platform and its SRC20 standard for security tokens. Swarm is a private blockchain that uses the Stellar open-source protocol, and its standard includes a voting process for token holders. SRC20 tokens can be traded on the Swarm platform. They are said to be interoperable across other security token exchanges, but so far, the number of tokens listed to verify is too limited. To illustrate this, Open Finance Network, one of the main security token exchange platforms, currently offers a meager four tokens for purchase; on the Swarm platform, none is available.

\section{Trading platforms that list security tokens}

To date, it seems that only a few security tokens, among those that are no longer locked, have already been listed; between those, great disparities remain.

For example, NEXO is listed on several exchange platforms and is traded daily in large volumes. But when it comes to real equity tokens, there are barely any listed or available for sale. For example, Mt Pelerin shares, whose tokens have been unlocked since February, are still awaiting to be listed. As a result, NEXO tokens are already being traded almost 50,000 times in a day, while only a few hundred Mt Pelerin token transfers can be found.

Other security tokens such as tZERO, Lottery.com and 22X Fund are only available for trade between accredited investors. Therefore, to comply with US regulations, their tokens are locked in wallets for 90 days. After this lock-up period, token holders may be able to resell them to other accredited investors but, 
apart from this option, their tokens are not freely tradeable for one year. As a result, most of these tokens won't be fully available for trading before the end of 2019.

Share registration processes and regulations, in many countries, are not always compatible with current exchange platforms such as Coinbase, Kraken or Binance. Indeed, such platforms should be able to handle dividend payments and to give proof of property to holders of security tokens so that they can vote; they should also enable easy withdrawal of security tokens, even when they have been acquired on another platform.

For those reasons, the trading of security tokens requires specialized portals such as Swarm, OpenFinance, Harbor, tZERO and Securitize. Other promising platforms are under development, like Ledgity. Yet, even the so-called operational platforms are still in their beta version, while most security tokens are still in their lock-up period.

Thus, our technical analysis shows that, as for ICOs, the vast majority of STOs rely on guidelines to create their tokens. However, unlike ICOs, there are only a few platforms that enable their trade, and most of these tokens remain solely available to accredited investors. Therefore, it is difficult to predict whether security tokens will be as successful as utility tokens. Indeed, as long as we have limited access to ergonomic platforms adapted to the exchange of security tokens, it is unlikely that the latter will be actively traded.

Nevertheless, blockchain actors are currently working hard to make this future market as liquid as possible. They are also followed by operators of current stock exchanges, like Boston, Gibraltar, the SIX Swiss Exchange-to only name a few-who plan to launch their own trading services for blockchain security tokens.

\subsection{Limitations on the Possibilities of Empirical Analysis}

During our research, it became apparent that it was almost impossible to carry out a relevant empirical analysis on trading volumes and prices of security tokens. Indeed, only four security tokens are currently listed on the OpenFinance security platform, one of the leader platforms. In addition, the tZERO security token, arguably the most renowned one at the moment, remains reserved for accredited investors, just like the tokens available on the OpenFinance platform. As a result, the number of investors registered on specialized platforms is very limited.

The only security tokens being currently traded are NEXO and Jinbi; however, they are not subject to regulatory constraints as important as the others, and they do not confer the same rights on shares or dividends. Nevertheless, we took a look at these two tokens that could be listed on traditional crypto exchange platforms.

For the NEXO token, we recorded $11,612,296,881$ tokens traded for $1,000,000$ tokens issued, giving an annual token turnover ratio of 12.5 , which is very high. 
On the other hand, the review of the Jinbi token led us to a different result. The token has been traded for 48 days only, and we recorded a total of 11,974 tokens exchanged. Combined with the 12,500,000 issued tokens, we achieved an annual turnover ratio of 0.007 . In this case, the liquidity is very low. To illustrate the meaning of this result, according to the World Bank, the average turnover ratio of major stock exchanges in the world was 35 in 2017. The highest value was in China (197.12), and the lowest value was in Luxembourg (0.12). According to the Economic Research of the Federal Reserve Bank of St. Louis, available on its website, the stock market turnover ratio (Value traded/Capitalization) in the US was around 130 in 2019.

We believe that the publication of comprehensive data on unlocked security tokens by the end of 2019, as well as the release of several platforms for their trading, will enable a useful complementary analysis on the blockchain-based equity possibilities.

\section{Conclusion and Discussion}

Liquidity is often cited as the most important factor in equity financing. Because the lack of liquidity limits the participation of investors and therefore increases the cost of capital, it is a determining element in the attractiveness of a market. This is currently the major problem faced by the SME stock market; for this reason, it is essential that alternative and competitive financing solutions emerge for this category of market players.

Our research has shown that while blockchain technology offers them a unique opportunity to raise equity more efficiently and cheaply than today, the success of STOs will largely depend on 1) the quality of their issuers and the opportunities of their market, 2) the existence of specialized platforms accessible to a wide range of investors and 3) the use of standards for security tokens in order to guarantee their interoperability across all these platforms. If these conditions are met, security tokens will most likely have the current success of the NEXO token, with significant trading volumes.

Our analysis also shows that there is a clear and solid presence of blockchain actors willing to build common standards to make this future market liquid. However, there is still a lot of opportunism from several entrepreneurs in this sector, and this could discourage investors in the same way it did for ICOs. Indeed, according to Conley [22], token offering that presents a lack of information increases investor uncertainty and perceived risk. As a result, such investors normally expect to be compensated for risk "and the willingness to pay for a share of profits is lower than it might be otherwise. This is exactly why the firms involved in IPOs try to be as clear and convincing about their prospects as possible". Likewise, the success of STOs and the liquidity of the security token market will depend on the quality of the issuers and of the information they provide to the public.

For the time being, companies involved in this new type of fundraising process remain specialists in the crypto market, making it difficult to anticipate 
its use by traditional SMEs. In addition, the trading market for security tokens is still immature. However, these new types of instruments have many characteristics that play in their favor, potentially making them a very competitive alternative to traditional equity financing. Therefore, there is a real need and opportunity for complementary empirical research to better understand this nascent market, but such work might not be feasible before several months or years, and at the very least not until most current security tokens are unlocked and fully available for trading.

\section{Conflicts of Interest}

The author declares no conflicts of interest regarding the publication of this paper.

\section{References}

[1] Cusmano L. and Koreen M. (2015) New Approaches to SME and Entrepreneurship Financing: Broadening the Range of Instruments. OECD Centre for Entrepreneurship, SMEs and Local Development.

[2] Nakamoto, S. (2008) Bitcoin: A Peer-to-Peer Electronic Cash System.

[3] Tapscott, D. (2017) How the Blockchain Is Changing Money and Business. TED Talk.

[4] Belleflamme, P., Lambert, T. and Schwienbacher, A. (2010) Crowdfunding: An Industrial Organization Perspective.

[5] Forbes, H. and Schaefer, D. (2017) Guidelines for Successful Crowdfunding. Procedia CIRP, 60, 398-403. https://doi.org/10.1016/j.procir.2017.02.021

[6] Downes, L. and Nunes, P. (2014) Big Bang Disruption: Strategy in the Age of Devastating Innovation. Penguin, London.

[7] Signori, A. and Vismara, S. (2016) Returns on Investments in Equity Crowdfunding. https://doi.org/10.2139/ssrn.2765488

[8] Keynes, J.M. (1930) Treatise Oil Money. Macmillan, London.

[9] Financial Conduct Authority (2013) The FCA's Regulatory Approach to Crowdfunding (and Similar Activities). FCA Consultation Paper CP13/13.

[10] (2015) DIY Crowdfunding White Paper. Quilageo, Inc.

[11] OECD (2014) New Approaches to SME and Entrepreneurship Finance: The Case of Crowdfunding. OECD, Paris.

[12] Unit, E.I. (2018) Capital Markets in 2030: The Future of Equity Capital Markets.

[13] Oliver Wyman, W.F.E. (2017) Enhancing Liquidity in Emerging Market Exchanges. OMFIF, Barclays.

[14] Ernst and Young Report (2009) IPO Insights Comparing Global Stock Exchanges.

[15] Peterhoff, D., Romeo, J. and Calvey, P. (2014) Towards Better Capital Markets Solutions for SME Financing. Oliver Wymann, New York.

[16] Buterin, V. (2013) Ethereum White Paper. GitHub Repository, 22-23.

[17] Zhu, H. and Zhou, Z. (2016) Analysis and Outlook of Applications of Blockchain Technology to Equity Crowdfunding in China. Financial Innovation, 2, 29. https://doi.org/10.1186/s40854-016-0044-7

[18] Swan, M. (2015) Blockchain: Blueprint for a New Economy. O’Reilly Media, Inc., 
Sebastopol.

[19] Chuen, L.D.K. and Linda, L. (2018) Inclusive FinTech: Blockchain, Cryptocurrency and ICO. World Scientific, Singapore.

[20] Finma (2018) Guidelines for Enquiries Regarding the Regulatory Framework for Initial Coin Offerings (ICOs).

[21] Vogelsteller, F. (2018) ERC: Token Standard \#20. Github.com. https://github.com/ethereum/eips/issues/20

[22] Conley, J.P. (2017) Blockchain and the Economics of Crypto-Tokens and Initial Coin Offerings (No. 17-00008). Vanderbilt University, Department of Economics, Nashville.

[23] Ante, L. and Fiedler, I. (2019) Cheap Signals in Security Token Offerings. https://doi.org/10.2139/ssrn.3356303

[24] Copeland, T. (2019) Decrypt Guide: What Is a Security Token and What Are STOs? Decrypt Media. https://decryptmedia.com/5311/security-token-offerings-advantages

[25] Mills, D., Wang, K., Malone, B., Ravi, A., Marquardt, J., Chen, C., Badev, A., Brezinski, T., Fahy, L., Liao, K., Kargenian, V., Ellithorpe, M., Ng, W. and Baird, M. (2016) Distributed Ledger Technology in Payments, Clearing, and Settlement. https://doi.org/10.21314/JFMI.2018.095

[26] Brunnermeier, M.K. and Pedersen, L.H. (2008) Market Liquidity and Funding Liquidity. The Review of Financial Studies, 22, 2201-2238. https://doi.org/10.1093/rfs/hhn098

[27] SEC (2017a) U.S. Securities and Exchange Commission. Investor Bulletin: Initial Coin Offerings.

https://www.sec.gov/oiea/investor-alerts-and-bulletins/ib_coinofferings

[28] Adhami, S., Giudici, G. and Martinazzi, S. (2018) Why Do Businesses Go Crypto? An Empirical Analysis of Initial Coin Offerings. Journal of Economics and Business, 100, 64-75. https://doi.org/10.1016/j.jeconbus.2018.04.001

[29] Fisch, C. (2019) Initial Coin Offerings (ICOs) to Finance New Ventures. Journal of Business Venturing, 34, 1-22. https://doi.org/10.1016/j.jbusvent.2018.09.007

[30] Ofir, M. and Sadeh, I. (2019) ICO vs IPO: Empirical Findings, Market Frictions and the Appropriate Regulatory Framework. Market Frictions and the Appropriate Regulatory Framework (February 19, 2019).

[31] Howell, S.T., Niessner, M. and Yermack, D. (2018) Initial Coin Offerings: Financing Growth with Cryptocurrency Token Sales (No. w24774). National Bureau of Economic Research, Cambridge. https://doi.org/10.3386/w24774

[32] Aouadi, A., Arouri, M. and Roubaud, D. (2018) Information Demand and Stock Market Liquidity: International Evidence. Economic Modelling, 70, 194-202. https://doi.org/10.1016/j.econmod.2017.11.005

[33] O’Neal, S. (2019) Who Scales It Best? Inside Blockchains. Ongoing TransactionsPer-Second Race. CoinTelegraph.

[34] Dossa, A., Ruiz, P., Vogelsteller, F. and Gosselin, S. (2018) ERC 1400: Security Token Standard. https://github.com/ethereum/eips/issues/1411 